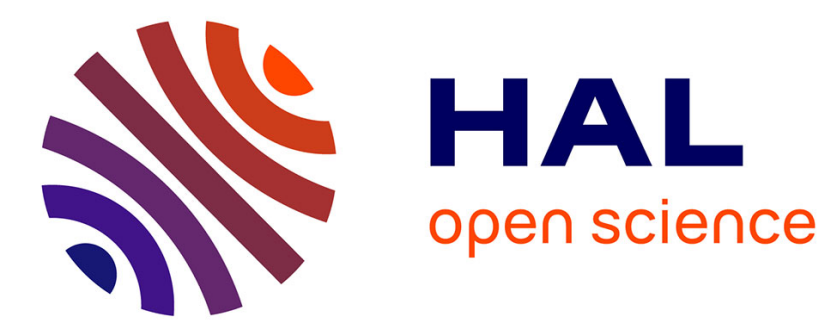

\title{
Dynamics of a Lyotropic Cubic Phase
}

Marianne Clerc, Yolande Hendrikx, Bela Farago

\section{To cite this version:}

Marianne Clerc, Yolande Hendrikx, Bela Farago. Dynamics of a Lyotropic Cubic Phase. Journal de Physique II, 1997, 7 (9), pp.1205-1214. 10.1051/jp2:1997181 . jpa-00248507

\section{HAL Id: jpa-00248507 https://hal.science/jpa-00248507}

Submitted on 1 Jan 1997

HAL is a multi-disciplinary open access archive for the deposit and dissemination of scientific research documents, whether they are published or not. The documents may come from teaching and research institutions in France or abroad, or from public or private research centers.
L'archive ouverte pluridisciplinaire HAL, est destinée au dépôt et à la diffusion de documents scientifiques de niveau recherche, publiés ou non, émanant des établissements d'enseignement et de recherche français ou étrangers, des laboratoires publics ou privés. 


\title{
Dynamics of a Lyotropic Cubic Phase
}

\author{
Marianne Clerc $\left({ }^{1, *}\right)$, Yolande Hendrikx $\left({ }^{1}\right)$ and Bela Farago $\left({ }^{2}\right)$ \\ $\left({ }^{1}\right)$ Laboratoire de Physique des Solides, Bâtiment 510, Université Paris-Sud, \\ 91405 Orsay, France \\ $\left({ }^{2}\right)$ Institut Laue Langevin, 38042 Grenoble cedex, France
}

(Received 17 February 1997, accepted 27 May 1997)
PACS.61.12.-q - Neutron diffraction and scattering
PACS.61.30.Eb - Experimental determinations of smectic, nematic, cholesteric, and other structures

\begin{abstract}
We study the cubic phase made of the non-ionic surfactant $\mathrm{C}_{12}(\mathrm{EO})_{6}$ in presence of water. The 3D periodic structure of this phase is characterized by the space group Ia3d. This structure corresponds to a centred cubic phase where the surfactant molecules build two identical 3D periodic interwoven labyrinths separated from each other by a continuous water medium. We present recent results obtained at I.L.L. on the Spin Echo spectrometer IN 11. Our experiments show for the first time, the dynamical character of the fluctuations observed in the investigated cubic phase. Therefore measurement have been performed on a single crystal $\left(\sim 0.1 \mathrm{~cm}^{3}\right)$. The time dependent diffusion function $S(\mathbf{q}, t)$ has been measured for different scattering vectors $\mathbf{q}$. For $\mathbf{q}$ vectors which do not correspond to Bragg peaks, the neutrons are scattered according to a quasi-elastic process and the associated $S(\mathbf{q}, t)$ function decreases with a characteristic time of about $20 \mathrm{~ns}$. The dynamical fluctuations are most probably related to collective deformation modes of the labyrinths.

Résumé. - Nous étudions la phase cubique du système binaire $\mathrm{C}_{12}(\mathrm{EO})_{6} /$ eau. La structure périodique tridimensionnelle de cette phase correspond au groupe d'espace Ia3d. Dans ce cas, la phase cubique est centrée et les molécules de surfactant s'organisent en deux labyrinthes tridimensionnels interpénétrés, séparés par un milieu continu d'eau. Nous présentons des résultats récents obtenus en utilisant le spectromètre spin-écho IN 11 à l'I.L.L. Nos expériences ont permis de mettre en évidence, pour la première fois, le caractère dynamique des fluctuations observées dans la phase cubique étudiée. Pour ce faire, des mesures ont été effectuées sur un monocristal $\left(\sim 0,1 \mathrm{~cm}^{3}\right)$. La fonction de diffusion $S(\mathbf{q}, t)$ a été mesurée pour différents vecteurs de diffusion $\mathbf{q}$. Pour des vecteurs q qui ne correspondent pas à des réflexions de Bragg, la diffusion des neutrons est quasi-élastique et la fonction associée, $S(\mathbf{q}, t)$, décroît avec un temps caractéristique d'environ 20 ns. Les fluctuations dynamiques sont probablement apparentées à des modes collectifs de déformation des labyrinthes.
\end{abstract}

This paper is the last contribution of Yolande Hendrikx, who departed from this life on March, the 28th 1997. We would like to dedicate this article to her memory.

$\left(^{*}\right)$ Author for correspondence (e-mail: clerc@lps.u-psud.fr)

(C) Les Éditions de Physique 1997 

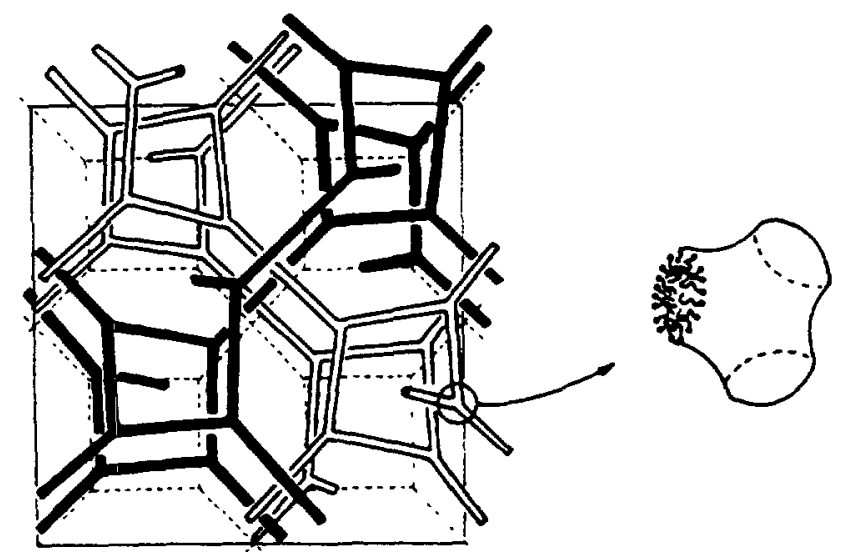

Fig. 1. - The direct bicontinuous cubic phase of space group Ia3d [6]. The surfactant molecules are building two equivalent infinite periodic 3D-labyrinths, one drawn with black lines, the other with white lines. The surfactant rods meet three by three at the vertices of the labyrinths. The two labyrinths are separated by an infinite periodic water film, that can be described using the Schoen Gyroid I.P.M.S. (this water film is not represented here). Two cubic unit cells are drawn along the vertical axis. The unit cell parameter, $a$, equals $110 \AA$ in the case of our $\mathrm{C}_{12} \mathrm{EO}_{6} /$ water sample.

\section{Introduction}

Bicontinuous cubic phases of surfactants or lipids in presence of water are now well-described [1-4]. They are direct or inverse and their extension domain (concentration and temperature ranges) as well as their location in phase diagrams depend on the involved surfactant molecules. At the macroscopic level, they are very viscous and optically isotropic. Their microscopic structure can be determined mainly using X-ray scattering or in some cases by image-filtering analyzes of freeze-fracture electron micrographs. A 3D-periodic order, characterized by a cubic unit cell, which results from the segregation of the surfactant molecules in water, is observed. In the case of a direct bicontinuous cubic phase, a water film separates two equivalent 3D-labyrinths formed by the so-called surfactant "rods" (see Fig. 1). In the symmetrical situation of an inverse bicontinuous cubic phase, the lipid molecules are building an infinite 3D-periodic fluid bilayer that separates two equivalent continuous water media. In both cases, the continuous medium (water film or surfactant bilayer) can be described using an infinite periodic minimal surface I.P.M.S. [3, 4]. Several cubic space groups are known (Ia3d, Pn3m, Im3m), corresponding to three different I.P.M.S. (the Schoen Gyroïd, the Schwarz F surface, the Schwarz $\mathrm{P}$ surface); the respectively associated 3D-labyrinths are formed by rods with different connectivities (3,4 and 6). In the case of the Ia3d cubic phase, shown in Figure 1, the connectivity of each system of rods is 3 .

These fascinating structures are of course not solid like, because both the water molecules and the surfactant molecules are in the liquid state. But they also exhibit a true 3D long range order at the mesoscopic scale. So one expects an unusual dynamical behaviour, as shown for instance by some theoretical predictions [5]. To our knowledge, we describe in this paper the first experiment that gives access to the dynamical fluctuations of a bicontinuous cubic phase.

We used the binary mixture of the non-ionic surfactant $C_{12} E_{6}$ with water, which exhibits a Ia3d direct bicontinuous cubic phase (see Fig. 1). This system has been extensively studied 
[6-8], because large single domains of the cubic phase can grow quite easily [8]. It is then possible to do an X-ray scattering experiment on a single cubic "crystal" [6,7]. X-ray patterns reveal intense diffuse scattering streaks in-between some Bragg peaks, showing that fluctuations of the average structure take place. These fluctuations are inherent to the cubic phase as they are observed far from any phase transformation. In order to elucidate the nature of these fluctuations, static defects or dynamical deformations, we decided to realize a neutron scattering experiment using the Neutron Spin Echo spectrometer at I.L.L., Grenoble. First, we will describe how we obtained a sufficiently large single cubic domain. Then, we will give the analysis of the scatterings of this single domain thanks to the Neutron Spin Echo technique.

\section{Experimental}

2.1. Sample Preparation. - In order to obtain the best signal to noise ratio required for our neutron experiments, $\mathrm{C}_{12} \mathrm{EO}_{6}$ molecules with perdeuterated alkyl chains are needed. Therefore such molecules were specially synthesized [9]. The deuteration of the alkyl chain appears to affect only very slightly the concentration range and the temperature range where the cubic phase is identified. The cubic phase has been prepared by mixing the $\mathrm{C}_{12} \mathrm{EO}_{6}$ molecules, either with $\mathrm{D}_{2} \mathrm{O}$, or a mixture of $\mathrm{H}_{2} \mathrm{O}$ and $\mathrm{D}_{2} \mathrm{O}$ which scattering length density matches that of the polar heads. The experiments which we are dealing with, concern the cubic phase prepared with such a water mixture. In order to match the scattering length density of the polar heads one must take into account the isotopic exchange between $\mathrm{H}$ and $\mathrm{D}$ which occurs for the terminal alcohol group of the polar heads and the isotopic purity of the deuterated water we used $(99.98 \%)$. We thus prepared a water mixture of $\mathrm{H}_{2} \mathrm{O}$ and $\mathrm{D}_{2} \mathrm{O}$, with a molar ratio $\mathrm{H}_{2} \mathrm{O} / \mathrm{D}_{2} \mathrm{O}=4.42$. The calculated scattering length densities are then, $0.679 \times 10^{10} \mathrm{~cm}^{-2}$ for water and $0.687 \times 10^{10} \mathrm{~cm}^{-2}$ for the polar heads. That of the deuterated alkyl chains is equal to $7.02 \times 10^{10} \mathrm{~cm}^{-2}$ and we may infer that when the perdeuterated $\mathrm{C}_{12} \mathrm{EO}_{6}$ molecules are mixed with the above water mixture only the alkyl chains are labelled for the neutron scattering. According to its composition, the molar ratio of our sample was, $\left(\mathrm{H}_{2} \mathrm{O}+\mathrm{D}_{2} \mathrm{O}\right) / \mathrm{C}_{12} \mathrm{EO}_{6}=14.60$. When equilibrium is achieved, the sample is homogeneous and forms a cubic phase at $20^{\circ} \mathrm{C}$.

At the above concentration, the following phase sequence versus temperature is observed [7]:

$$
\text { isotropic phase } \stackrel{70^{\circ} \mathrm{C}}{\longrightarrow} \text { lamellar phase } \stackrel{24^{\circ} \mathrm{C}}{\longrightarrow} \text { cubic phase } \stackrel{13^{\circ} \mathrm{C}}{\longrightarrow} \text { hexagonal phase. }
$$

For our neutron experiments, we used a square quartz cell $\left(30 \times 30 \mathrm{~mm}^{2}\right)$ with a thickness of $1 \mathrm{~mm}$. To fill it, the mixture was first heated in the isotropic phase and then rapidly introduced inside the cell. The cell was closed by Teflon plugs and sealed with glue. In order to allow the growth of large single cubic domains inside the cell, the sample was cooled very slowly $\left(0.3^{\circ} \mathrm{C}\right.$ per hour) from the isotropic phase $\left(75^{\circ} \mathrm{C}\right)$ down to the cubic phase $\left(21^{\circ} \mathrm{C}\right)$. During this controlled cooling process, the cell was observed between crossed polarizers [10].

As already mentioned, the cubic phase is optically isotropic and appears homogeneously black in polarized light (see Fig. 2). When it is cooled below $13^{\circ} \mathrm{C}$, it transforms into an hexagonal phase, according the above phase sequence. The hexagonal phase is birefringent (uniaxial medium) and one can observe its texture which presents large homogeneous domains inside the cell (see Fig. 2). Our previous study of the cubic/hexagonal phase transition [7] together with this observation allowed us to infer precisely the location of cubic single domains.

The sample was finally kept in the cubic phase at $21{ }^{\circ} \mathrm{C}$ during several weeks until the neutron experiment has been performed. 


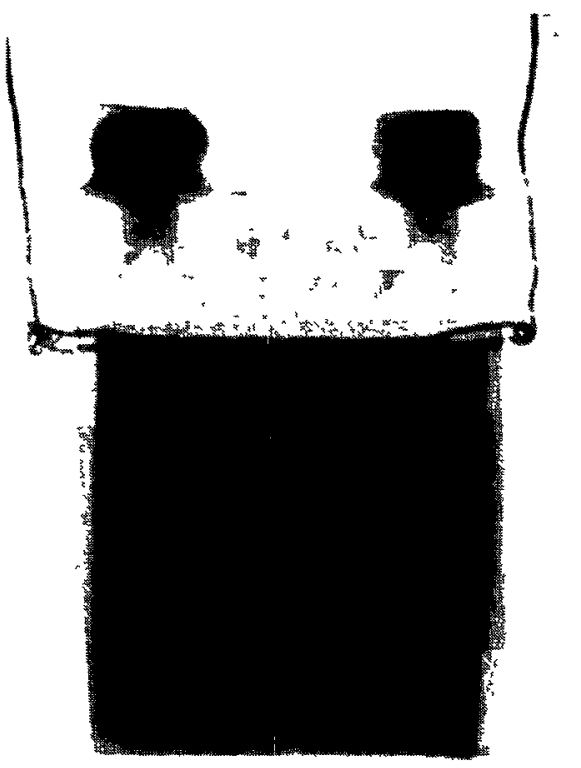

a)

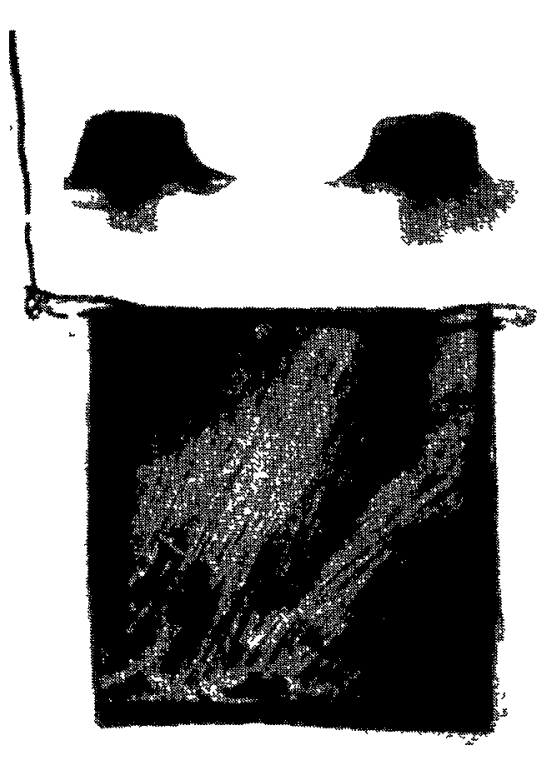

b)

Fig. 2. - Photographs of the cell in-between crossed polarizers a) $T=21{ }^{\circ} \mathrm{C}$. One observes the cubic phase, which is optically isotropic. The cell appears homogeneously black. b) $T=11^{\circ} \mathrm{C}$. One observes the hexagonal phase. The striated texture [7] allows to identify large homogeneous regions. The arrow indicates for instance the average orientation in the left upper part of the cell. In the lower part, small regions appear very dark, because their optical axes is almost perpendicular to the walls of the cell.

2.2. Small Angle Neutron Scattering. - The quality and the exact location of a single cubic domain was first checked by a small angle neutron scattering experiment (I.L.L., spectrometer D 17). The wavelength was equal to $\lambda=8.5 \AA(\Delta \lambda / \lambda=10 \%)$ and the neutron beam cross-section to $0.2 \mathrm{~cm}^{2}$. The spectrometer is provided with a $2 \mathrm{D}$-multidetector. The distance between the sample and the multidetector was $90 \mathrm{~cm}$.

The sample holder, thermostated at $21^{\circ} \mathrm{C}$ using a water bath circulation, was mounted on a goniometric head. The latter could be translated and different regions of the cell could be explored. When a large homogeneous region was detected, different rotations of the sample allowed to identify different orientations of the single cubic domain with respect to the cell walls.

Figure 3a shows a diffraction pattern of the single cubic domain we studied. Several Bragg peaks are observed, and in-between, intense diffuse streaks, as expected from the previous X-ray scattering studies $[6,7]$. Rocking-curves give an angular extension of $1.5^{\circ}$ for the Bragg peaks. This corresponds to a value of residual mosaicity comparable to the experimental resolution. We have verified that the positions in the reciprocal space of the Bragg peaks correspond to the diffraction due to a unique cubic domain. The sample was then translated with steps of $5 \mathrm{~mm}$ and we measured a size of about $1 \mathrm{~cm}^{2}$ for this domain, in agreement with our preliminary optical observations (see Sect. 2.1 or Fig. 2). This domain was then selected using 
a)

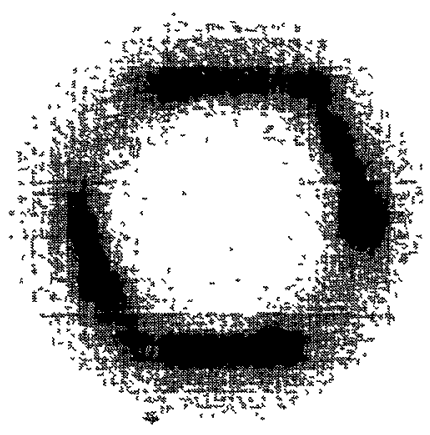

b)

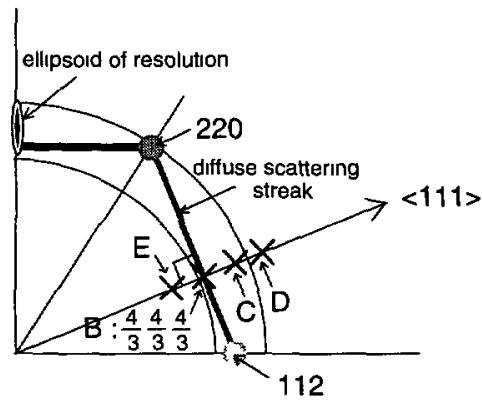

c)

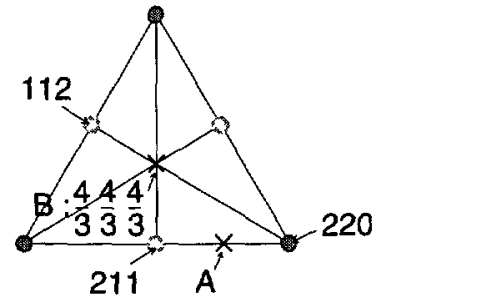

Fig. 3. - Small angle neutron scattering by the single cubic domain a) Pattern obtained on the 2D-multidetector at D 17 (I.L.L.). Single Bragg peaks are observed, all corresponding to (2 20 ) or $\left(\begin{array}{lll}2 & 1 & 1\end{array}\right)$ reticular planes of the cubic reciprocal lattice $(a=110 \AA)$. In-between the 220 and 112 Bragg peaks, a diffuse scattering streak is observed. b) Part of the reciprocal space observed in 3ax. One can see that the size of the ellipsoid of resolution allows to detect for a same $|\mathbf{q}|$, Bragg peaks corresponding to $\left(\begin{array}{lll}2 & 2 & 0\end{array}\right)$ or $\left(\begin{array}{lll}2 & 1 & 1\end{array}\right)$ reticular planes. The vector $\mathbf{q}=\frac{2 \pi}{a}\left(\frac{4}{3}, \frac{4}{3}, \frac{4}{3}\right) \AA^{-1}$ corresponds to the maximum intensity, $\mathrm{B}$, of the diffuse scattering along the $\left\langle\begin{array}{llll}1 & 1 & 1\end{array}\right)$ direction $[6,7]$. The three crosses $B, C$, and D correspond to the three $q$ vectors of Figures $5 b, 5 c$ and $5 d$. Point $E$ was also investigated (see Tab. I). c) Reciprocal plane perpendicular to the $\left\langle\begin{array}{llll}1 & 1 & 1\end{array}\right\rangle$ direction, where the diffuse scattering is located. Relative positions of A and B.

a cadmium mask so that the scattering area available for the neutron spin echo experiment was about $0.7 \mathrm{~cm}^{2}$.

2.3. The Neutron Spin Echo Experiment. - Results of the study of the single cubic domain are given in Section 3. We will first summarize some experimental features of the neutron spin echo experiment. The method and the instrument are described in details elsewhere $[11,12]$.

A detector is movable in the horizontal plane in order to select the momentum transfer $|q|$. From the small angle scattering experiment, we know the crystallographic orientation of the cubic single domain with respect to the quartz cell. Using the goniometric head, we can analyze the scattering at different $q$ vectors of the reciprocal space.

We used polarized neutrons with $\lambda=8.1 \AA$ and $\Delta \lambda / \lambda=18 \%$ FWHM monochromatization. The angular divergence of the beam before the sample is about 0.5 degree and after the sample 1.0 degree, as determined by round diaphragms. This gives a relatively bad $|q|$ resolution (18\%) along the $\mathbf{q}$ scattering vector and a relatively good resolution in the perpendicular direction. This fact was taken into account by the so-called ellipsoid of resolution (see Fig. $3 \mathrm{~b}$ ). The energy resolution of the instrument was calibrated with a standard elastic scatterer.

In a neutron spin-echo experiment, the directly measured quantity is $S(\mathbf{q}, t) / S(\mathbf{q}, 0)$, the normalized time dependent intermediate scattering function. The time $t$ is varied by changing 

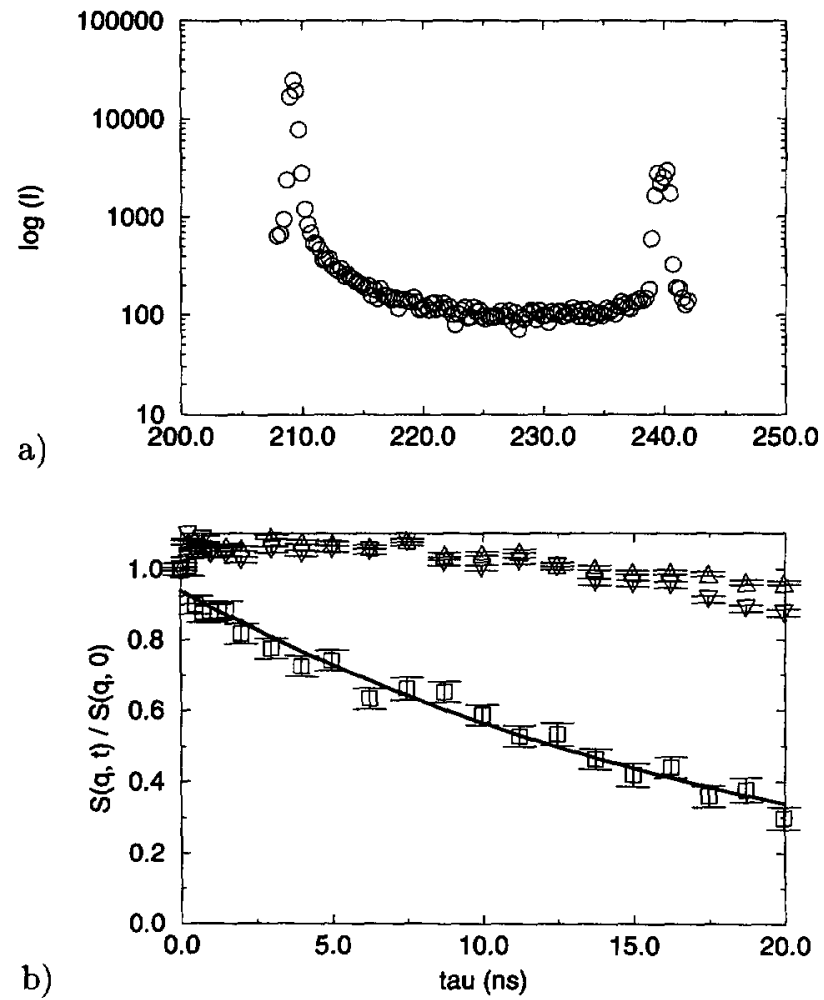

Fig. 4. - Comparison of the spin echo signal of a Bragg peak and of diffuse scattering. a) Intensity scan between the 211 and the 220 Bragg peaks (see Fig. 3c). As explained in Figure 3b, the size of the ellipsoid of resolution allows to detect these two Bragg peaks for a same $|q|$ value. The $X$ co-ordinate is the value of the angle of rotation of the cell around the vertical axis. In-between the two Bragg peaks, one measures the diffuse scattering contribution. b) $S(\mathbf{q}, t) / S(\mathbf{q}, 0)$ curves for the 211 Bragg peak, the 220 Bragg peak and point A. A mono-exponential decay time $\tau=19.6 \pm 0.7 \mathrm{~ns}$ is measured.

the field integral of the precession field $[11,13]$. In our case, the accessible time range was 0.25-20 ns. In the case of a pure elastic scattering, $S(\mathbf{q}, t) / S(\mathbf{q}, 0)$ does not decay but stays always equal to 1.0. If at least a fraction of the scattered intensity is quasi-elastic, with a time constant comparable to the instrumental window, this fraction of the observed signal will show some sort of decay.

\section{Results}

A suitable orientation of the cubic crystal was first checked performing an intensity scan by rotation of the sample around the vertical axis. The detector selects then a constant value of $|\mathbf{q}|$, chosen between $\left|\mathbf{q}_{211}\right|$ and $\left|\mathbf{q}_{220}\right|$. Indeed, the size of the ellipsoid of resolution (see Fig. 3b) allows to detect for this $|\mathbf{q}|$ value both Bragg peaks $\left(\left|\mathbf{q}_{220}\right|=\sqrt{\frac{8}{6}}\left|\mathbf{q}_{211}\right| \cong 1.155\left|\mathbf{q}_{211}\right|\right)$. Figure 4a shows the intensity scan at this $|\mathbf{q}|$ modulus and three $S(\mathbf{q}, t) / S(\mathbf{q}, 0)$ associated curves are shown in Figure $4 \mathrm{~b}$. The two sharp peaks of the intensity scan correspond to the 
(a) teta $/ 2$ teta scan and $10^{-9} /$ Deff

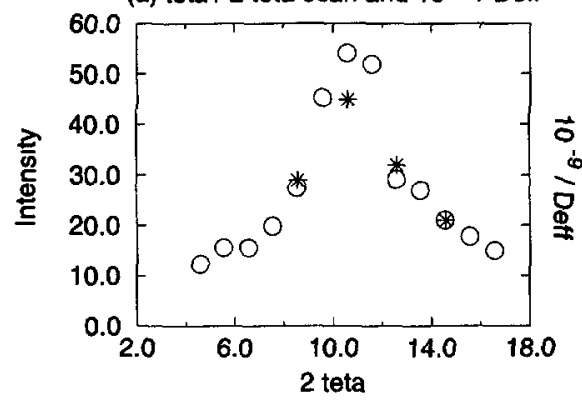

(d) $D: 2$ teta $=14.6$

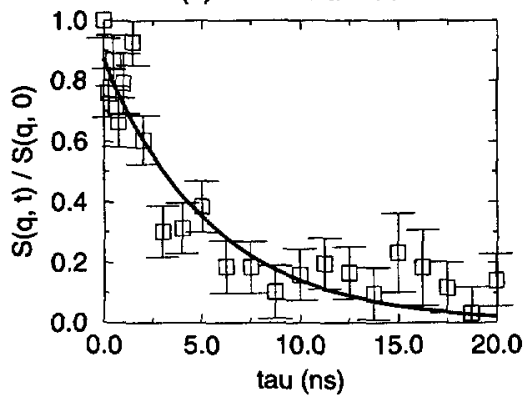

(b) $B: 2$ teta $=10.6$

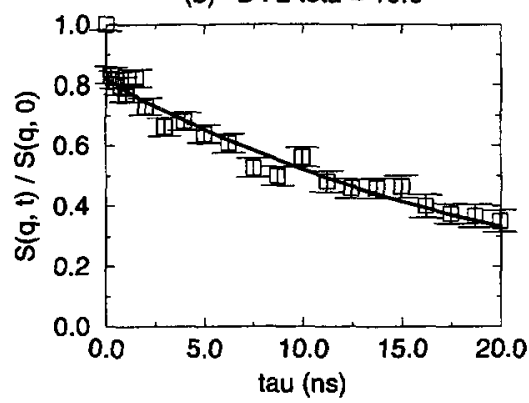

(c) $C: 2$ teta $=12.6$

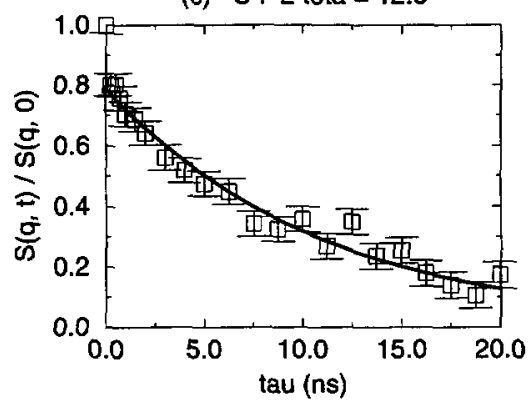

Fig. 5. - Spin echo signal along the $\left(\begin{array}{lll}1 & 1 & 1\end{array}\right)$ direction. a) $\theta / 2 \theta$ scan along the $\left\langle\begin{array}{lll}1 & 1 & 1\end{array}\right)$ direction. The maximum intensity of the diffuse scattering is measured for $2 \theta=10.6^{\circ}$, point $\mathrm{B}$, corresponding to the vector $\mathbf{q}=\frac{2 \pi}{a}\left(\frac{4}{3}, \frac{4}{3}, \frac{4}{3}\right) \AA^{-1}$ The stars correspond to $10^{-9} / D_{\text {eff }}\left(\mathrm{m}^{-2} \mathrm{~s}\right)\left(D_{\text {eff }}=\frac{1}{\tau q^{2}}\right.$, see Tab. I and Sect. 3). The intensity scale of the $\theta / 2 \theta$ scan has been adjust in order to show its similar behaviour to the one of $1 / D_{\text {eff }}$. b) $S(\mathbf{q}, t) / S(\mathbf{q}, 0)$ curve for $2 \theta=10.6^{\circ}$ (B). A mono-exponential decay time $\tau=22.1 \pm 1.1$ ns is measured. c) $S(\mathbf{q}, t) / S(\mathbf{q}, 0)$ curve for $2 \theta=12.6^{\circ}(\mathrm{C})$. A mono-exponential decay time $\tau=11.0 \pm 0.6 \mathrm{~ns}$ is measured. d) $S(\mathbf{q}, t) / S(\mathbf{q}, 0)$ curve for $2 \theta=14.6^{\circ}$ (D). A mono-exponential decay time $\tau=5.5 \pm 0.6 \mathrm{~ns}$ is measured.

211 and the 220 Bragg peaks. The $S(\mathbf{q}, t) / S(\mathbf{q}, 0)$ curves have been measured for $\mathbf{q}_{211}$, $\mathbf{q}_{220}$ and between both (point $\mathrm{A}$ in Fig. 4a), for $\frac{\mathbf{q}_{211}+\mathbf{q}_{220}}{2}=\frac{2 \pi}{a}(2,1.5,0.5) \AA^{-1}$ For the Bragg peaks, $S(\mathbf{q}, t) / S(\mathbf{q}, 0)$ is roughly equal to 1 at any time [14]. This means that the scattering originates from a purely elastic process. Contrariwise, the $S(\mathbf{q}, t) / S(\mathbf{q}, 0)$ curve at point A (Fig. 4a) decreases versus time. A quasi-elastic process is involved at this position, which corresponds to a diffuse scattering signal in-between the two Bragg peaks. Assuming a mono-exponential decay for $S(\mathbf{q}, t)=S(\mathbf{q}, 0) \exp (-t / \tau)$, we measure a decay time $\tau$ equal to $19.6 \pm 0.7 \mathrm{~ns}$.

We investigated a second direction of the reciprocal space, varying the value of the $|\mathbf{q}|$ modulus along the $\left\langle\begin{array}{lll}1 & 1 & 1\end{array}\right)$ axis (see Fig. $3 b$ ). No Bragg peak is crossed and only a diffuse scattering signal is measured. The $\theta / 2 \theta$ scan along the $\left\langle\begin{array}{lll}1 & 1 & 1\end{array}\right\rangle$ axis is shown in Figure $5 \mathrm{a}$. The maximum of intensity is obtained for $2 \theta=10.6^{\circ}$, at $q=\frac{2 \pi}{a}\left(\frac{4}{3}, \frac{4}{3}, \frac{4}{3}\right) \AA^{-1}[6,7]$. 
Table I. - Spin echo measurements along the $\left\langle\begin{array}{lll}1 & 1 & 1\end{array}\right)$ direction. For the four points we investrgated, $\tau$ is the time constant deduced from a mono-exponential fit. The quantity $D_{\text {eff }}=\frac{1}{\tau q^{2}}$ (Sect. 3) is calculated, in order to characterize furthermore the dependence of $\tau$ versus the $|\mathbf{q}|$ modulus.

\begin{tabular}{|c|c|c|c|c|}
\hline Echo points & $\mathrm{E}$ & $\mathrm{B}$ & $\mathrm{C}$ & $\mathrm{D}$ \\
\hline $2 \theta\left(^{\circ}\right)$ & 8.6 & 10.6 & 12.6 & 14.6 \\
\hline$|\mathbf{q}|\left(\AA^{-1}\right)$ & 0.116 & 0.143 & 0.17 & 0.197 \\
\hline$\tau(\mathrm{ns})$ & 21.5 & 22.1 & 11 & 5.5 \\
\hline$D_{\text {eff }} \times 10^{11} \mathrm{~m}^{2} \mathrm{~s}^{-1}$ & 3.46 & 2.21 & 3.15 & 4.68 \\
\hline $10^{-9} / D_{\text {eff }} \mathrm{m}^{-2} \mathrm{~s}$ & 29 & 45 & 32 & 21 \\
\hline
\end{tabular}

Three $S(\mathbf{q}, t) / S(\mathbf{q}, 0)$ curves are shown in Figure 5. They correspond to three values of $2 \theta$ on the $\theta / 2 \theta$ scan (Fig. 5a): $10.6^{\circ}$ (B), $12.6^{\circ}$ (C) and $14.6^{\circ}(\mathrm{D})$. The three curves are decreasing versus time. Again, we find that the scattering process is quasi-elastic when diffuse scattering is analyzed. Furthermore, the three curves do not extrapolate to 1 at $t=0$ (Fig. 5). This means that about $20 \%$ of the intensity decreases with a time constant faster than our first point at $0.24 \mathrm{~ns}$. This fast component can not be attributed to the scattering from the water. Indeed, solvent scattering is isotropic in this $|\mathbf{q}|$ range, while the diffuse scattering from the sample increases by a factor three between $2 \theta=14.6^{\circ}$ and $2 \theta=10.6^{\circ}$. This contribution can not come from the solvent, because its relative fraction would not remain always equal to about $20 \%$ for different $\mathbf{q}$ vectors.

The results concerning the slower decay are summarized in Table I, assuming again a monoexponential decay for $S(\mathbf{q}, t)$. On observes a strong dependence of the time constant $\tau$ versus the $|\mathbf{q}|$ modulus. In order to analyse this dependence, the quantity $D_{\text {eff }}=\frac{1}{\tau q^{2}}$ is calculated [15]. In the case of a pure diffusive process, $D_{\text {eff }}$ is a constant. Here, we observe that the variation of $1 / D_{\text {eff }}$ versus the $|\mathbf{q}|$ modulus is similar to the intensity profile of the diffuse scattering, as shown in Figure 5a. It means that $D_{\text {eff }}$ has a minimum at the maximum of the intensity profile. This looks similar to the de Gennes narrowing in liquids [16].

We have obtained two types of results: on one hand, the scattering process is purely elastic for a Bragg peak (Fig. 4), as expected for a true 3D long range order; on the other hand, the analysis of a diffuse scattering signal reveals always a slow quasi-elastic scattering process (Figs. 4 and 5), compatible with a single exponential decay. In addition to this slow decay, a fast component is involved within less than $0.24 \mathrm{~ns}$, that can not be attributed to the solvent.

Finally, it is worthwhile to compare the diffuse scatterings in A and B. They correspond to the maximum intensity of diffuse scattering along two different directions and they have approximately the same $|\mathbf{q}|\left(\left|\mathbf{q}_{\mathrm{A}}\right|=\frac{2 \pi}{a}|(2,1.5,0.5)| \AA^{-1}=\frac{2 \pi}{a} 2.55 \AA^{-1}\right.$ and $\left|\mathbf{q}_{\mathrm{B}}\right|=\frac{2 \pi}{a}\left|\left(\frac{4}{3}, \frac{4}{3}, \frac{4}{3}\right)\right| \AA^{-1}$ $=\frac{2 \pi}{a} 2.31 \AA^{-1}$ ). The measured $\tau$ values in $\mathrm{A}$ and in $\mathrm{B}$ are very close, i.e., $\tau_{\mathrm{A}}=19.6 \pm 0.7 \mathrm{~ns}$, and $\tau_{\mathrm{B}}=22.1 \pm 1.1 \mathrm{~ns}$. We may conclude that for any $q$ corresponding to the maximum intensity of a diffuse streak, the characteristic decay time is of the same order of magnitude, equal to $20 \mathrm{~ns}$. 


\section{Conclusion}

We succeeded to obtain a large enough single cubic domain essential for the spin echo experiment. Indeed, a large single domain reduces the count times and allows the analysis of weak scattering such as those of the diffuse scattering discussed in the present paper. All the $S(\mathbf{q}, t) / S(\mathbf{q}, 0)$ curves corresponding to diffuse scattering are decreasing versus time. This proves that dynamical fluctuations are at the origin of this diffuse scattering, observed before on the X-ray patterns $[6,7]$. In addition, the neutron spin echo experiment gives access to quantitative results about this dynamic, for instance the value of the time decay $\tau$ for a selected $\mathbf{q}$ vector. In the future, we will explore furthermore the dependence of $\tau$ versus the $|\mathbf{q}|$ modulus.

So far, our results concern a mixture where only the paraffinic chains are labelled. It seems us important to investigate samples with other contrasts, in particular a mixture where only the polar heads are labelled. Comparing these different samples, we expect to be able to separate different contributions to the dynamics. The sample we have already studied probe essentially the fluctuations of the structure, while a sample with only the polar heads labelled might show dynamics of the building-blocks themselves [15].

Most probably, collective modes of the building-blocks inside the cubic unit cell are involved. As already proposed [6,7], periodic rows of vertices of the surfactant rods, parallels to the $\left(\begin{array}{lll}1 & 1 & 1\end{array}\right)$ axes, should play a predominant role in the dynamics of a cubic Ia3d structure. Following this interpretation, each row (i) has an associated displacement $u_{(\imath)}$ along its direction. The displacements of the rows are assumed to be uncorrelated. From our N.S.E. experiment, we may conclude that the displacements are time-dependent $-u_{(\imath)}(t)-$ Then, it appears that $S(\mathbf{q}, t) / S(\mathbf{q}, 0)$ can be directly related to the correlation function versus time of the displacement of a row: $\left\langle u_{(\imath)}(0) u_{(\imath)}(t)\right\rangle_{(\imath)}$. The dependence of $\tau$ versus the $|\mathbf{q}|$ modulus could be then related to a correlation length for the displacement along a row.

Our experimental results should be interpreted in the frame of a complete dynamical model. However, the dynamics of a cubic phase is far to be entirely theoretically understood [5]. This study deserves being carried on, because the dynamical fluctuations we have studied are an inherent feature of the investigated cubic phase, and we may infer that they should play a role in the stability of this cubic phase.

\section{Acknowledgments}

We are specially grateful to F. Artzner (Laboratoire de Physique des Solides, Orsay), who has synthesized the surfactant molecule with a perdeuterated chain, in parallel to his PhD work, and to J.M. Goddard (Laboratoire de Physique des Solides, Orsay), for his conception of the temperature cooling device specially adjust for the neutrons quartz cell.

\section{References}

[1] Mariani P., Luzzati V. and Delacroix H. J. Mol. Biol. 204 (1988) 165.

[2] Fontell K., Colloid Polym. Sci. 268 (1990) 264.

[3] Charvolin J. and Sadoc J.F., J. Phys. France 48 (1987) 1559.

[4] Clerc M. and Dubois-Violette E., J. Phys. II France 4 (1994) 275. 
[5] Bruinsma R.F., J. Phys. II France 2 (1992) 425. In this reference, only the case of a Im3m bicontinuous cubic phase is considered, choosing the most simple symmetry group.

[6] Rancon Y. and Charvolin J., J. Phys. Chem. 92 (1988) 2646.

[7] Clerc M., Levelut A.M. and Sadoc J.F., J. Phys. II France 1 (1991) 1263-1276.

[8] Sotta P., J. Phys. II France 1 (1991) 763-772.

[9] Artzner F., Laboratoire de Physique des Solides, private communication.

[10] Goddard J.M., Laboratoire de Physique des Solides, private communication.

[11] F. Mezei, Ed., Neutron Spin Echo, Lecture Notes in Physics, Vol. 122 (Springer Verlag, 1980) p. 122

[12] "The Yellow Book", Guide to Neutron Research Falicities at the I. L. L., Grenoble (April 1994).

[13] The correspondence between the time $t$ and the precession field integral $\mathrm{H} \ell$ reads [11]: $t(\mathrm{~ns})=2.34 \times 10^{-7} \mathrm{H} \ell(\mathrm{A}) \lambda^{3}(\AA)^{3}$, where $\lambda$ is the average wavelength of the neutrons.

[14] In fact, $S(\mathbf{q}, t) / S(\mathbf{q}, 0)$ starts slightly above 1 and shows a slight drop. This is an instrumental artefact. On the one hand, the instrumental resolution was calibrated with a strong elastic scatterer which has a slowly decreasing scattered intensity as a function of $|\mathbf{q}|$. On the other hand, the Bragg peak of the sample i) remonochromatizes the beam according to the Bragg law, due to the relatively good angular resolution (this was experimentally checked) ii) will only partially fill the possible paths of the beam section which will probe different field inhomogeneities than the calibration sample. This problem happens only when measuring on a narrow Bragg peak. In between the Bragg peaks, on the diffuse scattering, none of the above mentioned problems show up.

[15] Farago B., Monkenbusch M., Goecking K.D., Richter D. and Huang J.S., Physica B 213 \& 214 (1995) 712-717.

[16] de Gennes P.G., Physica B 25 (1959). 